\title{
Jets at lowest mass accretion rates
}

\section{Dipankar Maitra ${ }^{1}$, Andrew Cantrell ${ }^{2}$, Sera Markoff ${ }^{3}$, Heino Falcke ${ }^{4}$, Jon Miller ${ }^{1}$, and Charles Bailyn ${ }^{2}$}

${ }^{1}$ Dept. of Astronomy, University of Michigan,

Ann Arbor, MI, USA 48109

email: dmaitra, jonmm@umich.edu

${ }^{2}$ Dept. of Astronomy, Yale University

New Haven, CT, USA 06511

email: andrew.cantrell, charles.bailyn@yale.edu

${ }^{3}$ Astronomical Institute "Anton Pannekoek", University of Amsterdam

1098 XH Amsterdam, The Netherlands

email: s.b.markoff@uva.nl

${ }^{4}$ Dept. of Astronomy, Radboud University

6500 GL Nijmegen, The Netherlands

email: H.Falcke@astro.ru.nl

\begin{abstract}
We present results of recent observations and theoretical modeling of data from black holes accreting at very low luminosities $\left(\mathrm{L} / \mathrm{L}_{\mathrm{Edd}} \lesssim 10^{-8}\right)$. We discuss our newly developed time-dependent model for episodic ejection of relativistic plasma within a jet framework, and a successful application of this model to describe the origin of radio flares seen in Sgr $\mathrm{A}^{*}$, the Galactic center black hole. Both the observed time lags and size-frequency relationships are reproduced well by the model. We also discuss results from new Spitzer data of the stellar black hole X-ray binary system A0620-00. Complemented by long term SMARTS monitoring, these observations indicate that once the contribution from the accretion disk and the donor star are properly included, the residual mid-IR spectral energy distribution of $A 0620-00$ is quite flat and consistent with a non-thermal origin. The results above suggest that a significant fraction of the observed spectral energy distribution originating near black holes accreting at low luminosities could result from a mildly relativistic outflow. The fact that these outflows are seen in both stellar-mass black holes as well as in supermassive black holes at the heart of AGNs strengthens our expectation that accretion and jet physics scales with mass.
\end{abstract}

Keywords. black hole physics, accretion, accretion disks, acceleration of particles, Galaxy: nucleus, radiation mechanisms: general

\section{Introduction}

Collimated relativistic outflows or "jets" are observed to be very closely associated with compact accretors like black holes and neutron stars where $G M / R c^{2} \lesssim 1$. Such jets are known to emit across a broad range of the electromagnetic spectrum, from radio to $\mathrm{X}$-rays, and perhaps even $\gamma$-rays. Sometimes jets can be imaged directly, but often their presence is inferred, such as from flat to slightly inverted spectral energy distribution (SED) in radio through IR (see, e.g., Markoff et al. 2001; Maitra et al. 2009a; Vila \& Romero 2010), correlation between fluxes (see, e.g., Gallo et al. 2003; Russell et al. 2010), and scaling relations connecting radio and X-ray luminosities with the black hole mass (Merloni et al. 2003; Falcke et al 2004; Gültekin et al. 2009).

In X-ray binaries (XRB), compact steady jets appear to turn off (on) as the source makes a transition from nonthermal to thermal (thermal to nonthermal) X-ray state, at luminosities typically a few percent of the Eddington luminosity. See e.g. Homan \& 
Belloni (2005), Remillard \& McClintock (2006) for details of X-ray states, and Fender (2006) for a review on XRB jets. However, there is no clear consensus as to any lower luminosity limit for jets, or even about the mode of accretion at low mass accretion rates $(\dot{M})$. While it is generally agreed that the radiative efficiency of the emitting plasma is low (e.g. Narayan \& Yi 1994; Blandford \& Begelman 1999) at low $\dot{M}$, it is not even clear whether the observed emission originates in an inflow or an outflow.

Obviously instrumental limitations become important at the lowest luminosities. Nevertheless, ever-improving technological advances are making it possible to detect sources at luminosities as low as $10^{-9} \mathrm{~L}_{\mathrm{Edd}}$, a limit which is constantly decreasing. Results of these fascinating experiments point strongly to the presence of outflows even at the lowest luminosities, for stellar as well as supermassive black holes. Here we present a case study of two such sources: Sagittarius A*, the supermassive black hole at the center of our galaxy, and the stellar mass X-ray binary A0620-00, both of which suggest the presence of a jet at luminosities $\lesssim 10^{-8} \mathrm{~L}_{E d d}$.

\section{Sagittarius A*}

Located $8.4 \pm 0.6 \mathrm{kpc}$ (Reid et al. 2009) away at the Galactic Center, Sagittarius A* (hereafter Sgr $\mathrm{A}^{*}$ ) is the nearest "supermassive" black hole with a mass of $\sim 4 \times 10^{6} \mathrm{M}_{\odot}$ (Ghez et al. 2008). Despite its relative proximity, the source of emission from Sgr $\mathrm{A}^{*}$ is still unknown. This is partly due to the extremely high extinction in the direction of the Galactic Center, and partly to the fact that with a bolometric luminosity $\sim 10^{-9} L_{\mathrm{Edd}}$, Sgr $A^{*}$ is extremely under-luminous compared to other active galactic nuclei (AGN). However, flares and data from multiwavelength campaigns provide important clues about the nature of emission from Sgr A*. We have developed a time-dependent jet model which for the first time allows comparing the model predictions with radio flare data from Sgr $A^{*}$. Taking into account relevant cooling mechanisms, we calculate the frequencydependent time lags and photosphere size and compare with recent observations (see Fig. 1). Details of the modeling is presented in Maitra et al. (2009b).
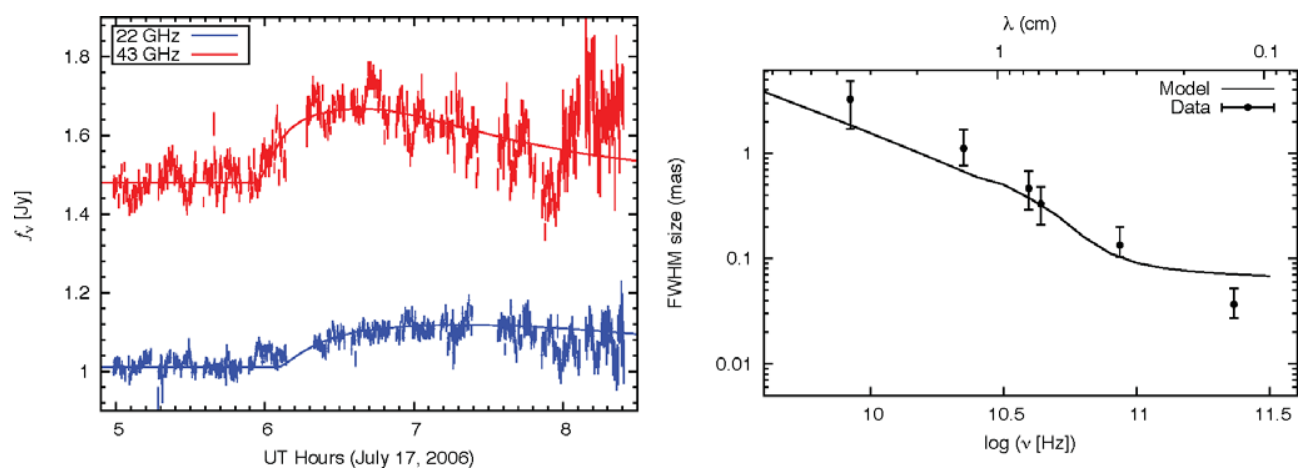

Figure 1. Left: Comparison of model with Sgr A* data for the flare on 2006 July 17 at 43 and $22 \mathrm{GHz}$. The $43 \mathrm{GHz}$ data with error bars from Yusef-Zadeh et al. (2008) are shown in red, and $22 \mathrm{GHz}$ data+model in blue. We model the flare that created a peak in the $43 \mathrm{GHz}$ light curve close to 6.5 hours UT. Right: Comparison of model-predicted frequency-size relationship (solid line) with observations. The data are from Bower et al. (2004), Shen et al. (2005), and Doeleman et al. (2008). Figures taken from Maitra et al. (2009b). 


\section{3. $\mathbf{A 0 6 2 0 - 0 0}$}

Since its massive outburst in 1975, A0620-00 has been in quiescence, with luminosities around $10^{-8} \mathrm{~L}_{\mathrm{Edd}}$. It has been detected in quiescence in X-rays, radio, IR, near-IR and optical using various space-based as well as ground-based instruments.

Despite easy detection in the optical and near-IR, estimating the mass of the compact accretor has been quite a challenge. An accurate (dynamical) measurement of the accretor mass requires correct knowledge of the orbital inclination. Historically attempts to measure the inclination from light curves have given widely varying results with $38^{\circ}<i<75^{\circ}$. However, recently Cantrell et al. $(2008,2010)$ have analyzed $\sim 30$ years of A0620-00 optical and near-IR (OIR) data and showed that previous disagreements on the inclination of A0620-00 were caused by (1) improper estimation of the disk flux (which can be significant even in quiescence), (2) nightly variability in light curve shape, and (3) not recognizing that even during quiescence, the OIR light curve shows three distinct optical states (named passive, loop, and active, and characterized by magnitude, color, and aperiodic variability). Of these three states the passive state is the faintest, shows consistent periodicity and is perhaps the closest to true quiescence. Consistent estimates of the binary parameters are obtained when the above effects are properly accounted for, and the best estimates of $(i, \mathrm{M})$ are $\left(51.0^{\circ} \pm 0.9^{\circ}, 6.6 \pm 0.25 \mathrm{M}_{\odot}\right)$. Accurate knowledge of the stellar contribution also allows determining any nonstellar residuals in the photometric data.

Here we present results of SMARTS long-term photometric monitoring of A062000 in OIR and a Spitzer/IRAC observation in 2006 November 26. Both the long-term monitoring as well as the Spitzer snapshot suggest the presence of nonthermal emission, presumably from a jet (see Fig. 2).
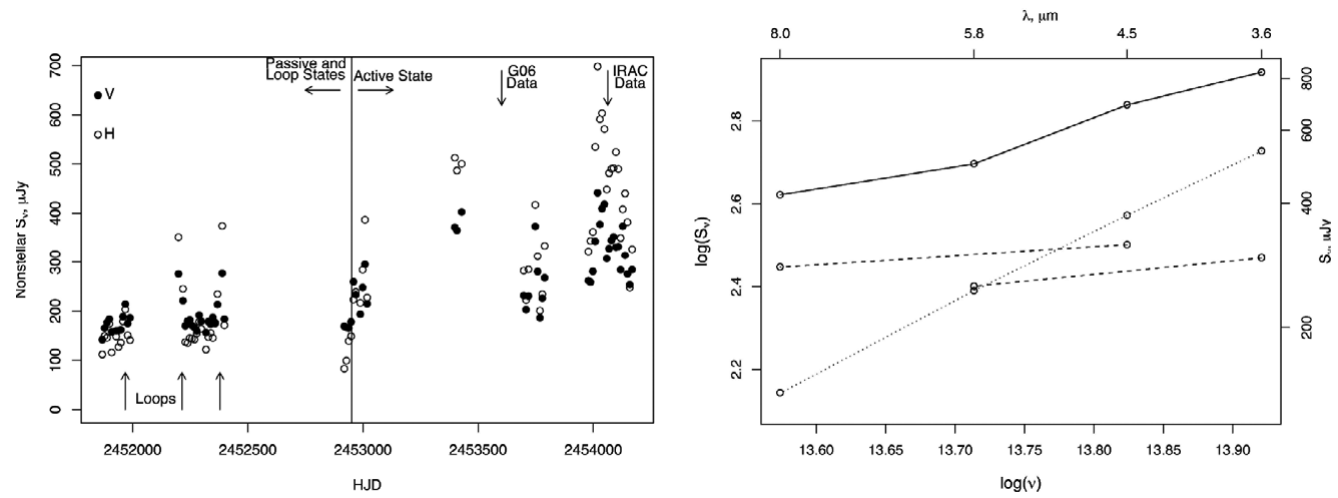

Figure 2. Left: Dereddened, nonstellar V- (filled points) and H-band (open points) flux densities from SMARTS monitoring of A0620-00. OIR state transitions as described in Cantrellet al.2010 are marked, as are the dates of the Spitzer/IRAC data and the Galloet al.(2006) data. A0620-00 is consistently redder in the active state than in the passive state, possibly due to a nonthermal contribution in the active state. Right: The solid line shows the IRAC SED of A0620-00. Also shown are the expected contributions from the donor + accretion disk (dotted line), and the residual "excess" (observed SED minus the donor+disk; dashed lines). The dashed lines connect the two pairs of bands which were obtained simultaneously. The excess has a relatively flat spectrum with slope $\sim 0.3$, suggesting that it could be at least partially nonthermal. 


\section{Discussion and Conclusions}

Recent multiwavelength data show that relativistic outflows or jets can be important at luminosities as low as $\sim 10^{-9} L_{\mathrm{Edd}}$, and hence affect the evolution and energetics of compact accretors. Here we have presented a case study of two low-luminosity accreting black hole sources, Sgr A* and A0620-00. The two sources differ by five orders of magnitude in mass, but still the data point toward the presence of an outflow in both.

In particular, for Sgr $\mathrm{A}^{*}$ we show that a time-dependent relativistic jet model can successfully reproduce:

- The quiescent broadband spectral energy distribution of Sgr A*,

- The observed 22 and $43 \mathrm{GHz}$ light curve morphologies and time lags,

- The frequency-size relationship.

These results suggest that the observed radio emission from Sgr A* is most easily explained by a stratified, optically thick, mildly relativistic jet. Frequency-dependent measurements of time-lags and intrinsic source size provide strong constraints on the bulk motion of the jet plasma.

For A0620-00, radio, Spitzer, and SMARTS data obtained during quiescence suggest:

- Significant excess above the expected thermal emission from donor + accretion disk; spectral index of the excess $-0.9<\alpha<-0.5\left(f_{\nu} \sim \nu^{\alpha}\right)$, i.e. consistency with optically thin jet synchrotron emission in the NIR,

- Nearly flat IR spectral index from Spitzer observations is suggestive of optically thick synchrotron emission in IR.

Techniques like Doppler tomograms of quiescent systems will lead to better understanding of gas flow in the accretion disc of A0620-00 and also that of the gas stream-disc impact point. Future VLBI, submm and time-lag measurements over a broader range of wavelengths will reveal greater details about ongoing physical processes within a few gravitational radii for sources like Sgr A* and M87.

\section{References}

Blandford, R. \& Begelman M. 1999, MNRAS, 303, L1

Bower, G. et al. 2004, Science, 304, 704

Cantrell, A. et al. 2008, ApJ, 673, L159

Cantrell, A. et al. 2010, ApJ, 710, 1127

Doeleman, S. et al. 2008, Nature, 455, 78

Falcke, H. et al. 2004, A\& $A, 414,895$

Fender, R. 2006, In "Compact Stellar X-ray Sources", Cambridge Astrophysics Series, 39, 381

Gallo, E. et al. 2003, MNRAS, 344, 60

Ghez, A. et al. 2008, ApJ, 689, 1044

Gültekin, K. et al. 2009, ApJ, 706, 404

Homan, J. \& Belloni, T. 2005, ApESSS, 300, 107

Maitra, D. et al. 2009a, MNRAS, 398, 1638

Maitra, D. et al. 2009b, A\& A, 508, L13

Markoff, S. et al. 2001, A\&AA, 372, L25

Merloni, A. et al. 2003, MNRAS, 345, 1057

Narayan, R. \& Yi I. 1994, ApJ, 428, L13

Reid, M. et al. 2009, ApJ, 700, 137

Remillard, R. \& McClintock J. 2006, ARAESA, 44, 49

Russell, D. et al. 2010, MNRAS, 405, 1759

Shen, Z.-Q. et al. 2005, Nature, 438, 62

Vila, G. \& Romero G. 2010, MNRAS, 403, 1457

Yusef-Zadeh, F. et al. 2008, ApJ, 682, 361 


\section{Discussion}

Benaglia: Could you think on radio polarization observations to confirm the nonthermal emission?

MAITRA: Not with instruments available at the time: for the radio source associated with A0620-00 is a $\sim 50 \mu \mathrm{Jy}$ source, and the expected polarization level is of a few $\%$. It might be feasible with the EVLA.

MirABEL: Some time delays at different wavelengths are observed in the ejecta of GRS $1915+105$, which implies that the time delays are determined by the adiabatic expansion of the plasma irrespective of the mass of the black hole. How do you get rid of the scattering to determine the frequency-size relation?

MAITRA: Yes, the VLT observations of Sgr $A^{*}$ have shown the existence of NIR flares. The NIR/X-ray flares trace particle (re)organization and cooling very close to the black hole, while marginally affecting the optically thick radio flux (that we modelled here). The presence of tge scattering screen in the direction of Sgr A* had indeed been the biggest obstacle towards obtaining the intrinsic size of Sgr A*. However, this problem has recently been overcome with the innovation of VLBI closure amplitude analysis (e.g. Doeleman et al. 2001). Closure amplitude method gives an accurate measurement of the source size. The scatter broadening follows a $\lambda^{2}$ law and this has been measured fairly accurately for Sgr A*, in turn giving good estimates of the intrinsic size of Sgr A*.

Russel: What is the spectral index of the optical-IR flares in quiscence of A0620?

Maitra: According to the preliminary done so far, equally good fits to all data can be obtained assuming any jet slope in the range $-0.9<\alpha<-0.5$, where $f_{\nu} \sim \nu^{\alpha}$.

MiLleR-Jones: What is the time resolution of the A0620-00 light curves and how finely can you bin them?

MAITRA: The minimum exposure time required to obtain good signal-to-noise for A062000 using the CTIO+SMARTS telescope s about 10 minutes. Right now we observe A0620 approximately once every night, but the number of observations per night could be increased (at least temporarily) if needed. 